\title{
Merging of vortices and antivortices in polariton superfluids
}

\author{
E. Cancellieri, ${ }^{1,2}$ T. Boulier, ${ }^{1}$ R. Hivet,${ }^{1}$ D. Ballarini, ${ }^{3}$ D. Sanvitto, ${ }^{3}$ M. H. Szymanska, ${ }^{4}$ C. Ciuti, ${ }^{5}$ \\ E. Giacobino, ${ }^{1}$ and A. Bramati ${ }^{1}$ \\ ${ }^{1}$ Laboratoire Kastler Brossel, Université Pierre et Marie Curie, Ecole Normale Supérieure et CNRS, UPMC case 74, \\ 4 place Jussieu, 75005 Paris, France \\ ${ }^{2}$ Department of Physics and Astronomy, University of Sheffield, Sheffield S3 7RH, United Kingdom \\ ${ }^{3}$ NNL, Istituto Nanoscienze-CNR, Via Arnesano, 73100 Lecce, Italy \\ ${ }^{4}$ Department of Physics and Astronomy, University College London, Gower Street, London WC1E 6BT, United Kingdom \\ ${ }^{5}$ Laboratoire Matériaux et Phénoménes Quantiques, UMR 7162, Université Paris Diderot-Paris 7 et CNRS, 75013 Paris, France
}

(Received 16 May 2014; revised manuscript received 15 October 2014; published 19 December 2014)

\begin{abstract}
Quantized vortices are remarkable manifestations on a macroscopic scale of the coherent nature of quantum fluids, and the study of their properties is of fundamental importance for the understanding of this peculiar state of matter. Cavity polaritons, due to their double light-matter nature, offer a unique controllable environment to investigate these properties. In this paper we theoretically investigate the possibility to deterministically achieve the annihilation of a vortex with an antivortex through an increase of the polariton density in the region surrounding the vortices. Moreover, we demonstrate that by means of this mechanism an array of vortex-antivortex pairs can be completely washed out.
\end{abstract}

DOI: 10.1103/PhysRevB.90.214518

PACS number(s): 71.36.+c, 03.75.Lm, 42.65.Hw

\section{INTRODUCTION}

Topological excitations such as quantized vortices, characterized by a phase winding from 0 to $2 \pi m$ (with $m$ an integer number) around a vortex core, have been extensively studied in several systems such as nonlinear optical systems [1], superconductors [2], superfluid ${ }^{4} \mathrm{He}$ [3], verticalcavity surface-emitting lasers [4], and, more recently, in cold atoms [5-7]. Finally, in recent years, the study of vortices and vortex lattices has attracted much attention also in the field of coherent cavity-polariton fluids, first, because, being intrinsically out of equilibrium, they constitute a novel system to study Bose-Einstein condensation phenomena, and second, because, due to their light-matter nature, polaritonic systems are fully controllable by optical techniques and therefore allow very detailed studies of quantum turbulence.

In particular, in the context of cavity-polariton systems, it has been shown that stable vortices and half vortices [8-10] as well as single vortex-antivortex (V-AV) pairs [11-14] can be generated. The formation of lattices of vortices and of vortex-antivortex pairs has also been theoretically and experimentally studied in several different configurations: in the optical parametrical oscillator configuration [15], in nonresonantly generated condensates [16-18], and in the case of patterns induced by metallic deposition on the surface of the cavity [19]. However, the mechanisms lying beneath vortex-antivortex annihilation and vortexvortex interactions are still not fully understood. This is related to the high degree of control needed to study such interactions.

For example, in the first experimental observations of vortex-antivortex lattices [17-19] the formation and the properties of the array were only partially controllable due to either the presence of a strong exciton reservoir, which influences the position of the formed vortex array and its disappearance, or due to the fact that the formed array depends on the structure of the metallic depositions over the cavity surface. To achieve a higher degree of control, resonant pumping schemes have been proposed [20]. By using masks in the pumping beam, the formation of vortex-antivortex arrays with controllable shapes and vortex distributions was achieved [21], and the evolution of the arrays was studied in correlation with the local onset of the superfluid regime. However, in this study the case of high-polariton densities was not experimentally achievable since the masks used to generate the vortex array were blocking most of the laser power, and therefore were not theoretically investigated.

In this paper we theoretically study the annihilation of a vortex with an antivortex when injecting polaritons resonantly, in a broad range of polariton densities. In our model we assume an excitation with four coherent laser beams resonant with the lower-polariton branch similarly to Refs. $[17,18]$ where, however, polaritons were injected nonresonantly. The four pump spots are supposed to have the same energy and $k$-vector modulus and to generate polaritons propagating toward the center of a common area. The advantage of our model is that in the resonant configuration it is well known that there is no exciton reservoir and that, since the entire pump intensity is used to inject the coherent fluid, high-polariton densities can be achieved. Moreover, since the pumps set the momentum and the density of the injected fluid, we can directly correlate the vortex-antivortex annihilation and the washing out of an array of vortices with an increase of the regions where the fluid is subsonic and with a change in the polariton flow that comes with it.

The paper is structured as follows: In Sec. II we introduce the theoretical model used to simulate the system, describe in more detail the setup we have in mind, and introduce the generalized local speed of sound that will be needed for the analysis of the results. In Sec. III we demonstrate the annihilation of V-AV pairs and correlate this annihilation with the change in the polariton flow caused by the widening of the regions where the fluid is subsonic. Finally, in Sec. IV we draw 
some conclusions and give prospects for future developments of the work.

\section{MODEL}

A standard way to model the dynamics of resonantly driven polaritons in a planar microcavity is to use a Gross-Pitaevskii (GP) equation [22] for coupled cavity and exciton fields $\left(\Psi_{C}\right.$ and $\Psi_{X}$ ) generalized to include the effects of the resonant pumping and decay $(\hbar=1)$ :

$$
\partial_{t}\left(\begin{array}{l}
\Psi_{X} \\
\Psi_{C}
\end{array}\right)=\left(\begin{array}{l}
0 \\
F
\end{array}\right)+\left[H_{0}+\left(\begin{array}{cc}
g_{X}\left|\Psi_{X}\right|^{2} & 0 \\
0 & V_{C}
\end{array}\right)\right]\left(\begin{array}{l}
\Psi_{X} \\
\Psi_{C}
\end{array}\right),
$$

where the single particle polariton Hamiltonian $H_{0}$ is given by

$$
H_{0}=\left(\begin{array}{cc}
\omega_{X}-i \kappa_{X} / 2 & \Omega_{R} / 2 \\
\Omega_{R} / 2 & \omega_{C}(-i \nabla)-i \kappa_{C} / 2
\end{array}\right),
$$

and

$$
\omega_{C}(-i \nabla)=\omega_{C}(0)-\frac{\nabla^{2}}{2 m_{c}}
$$

is the cavity dispersion, with the photon mass $m_{C}=5 \times$ $10^{-5} m_{0}$ and $m_{0}$ the bare electron mass. For our simulations we assumed a flat exciton dispersion relation $\omega_{X}(\mathbf{k})=\omega_{X}(0)$, set the exciton-photon detuning to zero, $\delta_{\text {ex-ph }}=\omega_{X}(0)-$ $\omega_{C}(0)=0$, and set this energy value as the reference of the zero energy. The parameters $\Omega_{R}, \kappa_{X}$, and $\kappa_{C}$ are the Rabi frequency and the excitonic and photonic decay rates, respectively, and have been given values close to the usual experimental ones: $\Omega_{R}=5.1 \mathrm{meV}, \kappa_{X}=0.05 \mathrm{meV}$, and $\kappa_{C}=0.08 \mathrm{meV}$ [21]. In this model polaritons are injected into the cavity by four coherent and monochromatic laser fields with pump intensity $f_{p}$ and Gaussian spatial profiles with $\sigma_{p}$ of $20 \mu \mathrm{m}: F(\mathbf{x})=$ $\sum_{i=1}^{4} f_{p} e^{i \mathbf{k}_{\mathbf{p i}} \mathbf{x}} e^{-\left(\mathbf{x}-\mathbf{x}_{\mathbf{i}}\right)^{2} / 2 \sigma_{p}^{2}}$, where $\mathbf{k}_{\mathbf{p}_{\mathbf{i}}}$ are the four wave vectors of the four pumps that we fix to have the same modulus, $\left|\mathbf{k}_{\mathbf{p}_{\mathbf{i}}}\right|=|\mathbf{k}|$. In order to ensure that the phase of the polariton fluid is not imposed by the laser pumps in the central region of the system, we set to zero the pump intensity outside of a $\sigma_{\text {pin }}=$ $9 \mu \mathrm{m}$ radius circle. The exciton-exciton interaction strength $g_{X}$ is set to one by rescaling both the cavity and excitonic fields and the pump intensities. The numerical solution of the GP equation is obtained over a two-dimensional grid (of $512 \times 512$ points) in a box with sides of $150 \times 150 \mu \mathrm{m}^{2}$ using a fifth-order adaptive-step Runge-Kutta algorithm. All the analyzed quantities are taken when the system has reached a steady state condition after a transient period of $200 \mathrm{ps}$.

To understand the role of the subsonic character of the fluid in the annihilation of a vortex-antivortex pair, we define the local fluid velocity $v_{f}(\mathbf{x})=\hbar|\mathbf{k}(\mathbf{x})| / m_{\mathrm{LP}}$, where $m_{\mathrm{LP}}$ is the lower-polariton mass and $\mathbf{k}(\mathbf{x})$ is the locally evaluated derivative of the phase at the point $\mathbf{x}$. Moreover, we define the quantity $c_{s}(\mathbf{x})=\sqrt{\hbar g_{\mathrm{LP}}\left|\Psi_{\mathrm{LP}}(\mathbf{x})\right|^{2} / m_{\mathrm{LP}}}$, where $\left|\Psi_{\mathrm{LP}}(\mathbf{x})\right|^{2}$ and $g_{\mathrm{LP}}$ are the local density and the polariton-polariton interaction constant. Since in the local density approximation $c_{S}(\mathbf{x})$ corresponds to the speed of sound defined in the case of high densities [23,24], we can take it as the definition of a generalized local speed of sound that is valid also for low-polariton densities and we define a generalized Mach number:

$$
M(\mathbf{x})=\frac{v_{f}(\mathbf{x})}{c_{s}(\mathbf{x})}=\frac{\hbar|k(\mathbf{x})| / m_{\mathrm{LP}}}{\sqrt{\hbar g_{\mathrm{LP}}\left|\Psi_{\mathrm{LP}}(\mathbf{x})\right|^{2} / m_{\mathrm{LP}}}} .
$$

This will allow one to establish a direct correlation between the subsonic or supersonic character of the fluid and the annihilation of a V-AV pair. Since the subsonic character of the fluid is induced by a polariton-polariton interaction, this corresponds to studying the role of polariton-polariton interactions in the annihilation of the pairs and of the vortex array. Although other techniques, such as the study of the vortex-antivortex correlation function [25] or Reynolds-averaged Navier-Stokes equations [26], can be used to address this problem, we chose to focus on the study of the Mach number since it allows a simple and clear physical understanding of the V-AV merging process.

\section{RESULTS}

The mechanism lying beneath the annihilation of $\mathrm{V}-\mathrm{AV}$ pairs and the role of polariton-polariton interactions in this annihilation can be better highlighted by studying the system behavior as a function of the pump intensity and therefore of the polariton density. We start by studying the system in the two limiting cases of very low and very high pump intensities.

In the low intensity case the polariton density lies on the lower branch of the bistability curve everywhere in space and the system behavior is purely linear. In this regime [Figs. 1(a)$1(c)]$, the formation of an array of vortices and antivortices is observed as in Refs. [17,18,21]. Here, as in Ref. [21], the shape and size of the unit cell only depend on the geometry of the pumping configuration and on the angle of incidence of the laser beams. Since we use four pumps with $|\mathbf{k}|=0.7 \mu \mathrm{m}^{-1}$, the formed array has square unit cells with a unit cell size of approximately $9 \mu \mathrm{m}$ [Figs. 1(a)-1(c)]. This interference pattern generates an array of vortices (with a clockwise phase winding from $-\pi$ to $\pi$ ) and antivortices (with an anticlockwise phase winding from $-\pi$ to $\pi$ ) that is therefore due to a purely linear mechanism [see Fig. 1(c) and the inset for the definition of vortices and antivortices].

In the opposite limit the pump intensity is strong and polariton-polariton interactions play a dominant role. This regime is characterized by a polariton density everywhere lying on the upper branch of the bistability curve. As can be seen in Figs. 1(d)-1(f), in this regime the array of vortices and antivortices has completely disappeared due to the renormalization of the lower-polariton branch.

From this, we can deduce that in the transition region between the low and the high density regimes the vortices and antivortices either are expelled from the fluid or annihilate each other. In order to investigate this transition, we vary the pump intensity around the threshold value $\left(f_{\text {th }}\right)$ at which the V-AV pairs disappear. Note that in this nonhomogeneous system the density is different in the regions within and outside the pumping spot, resulting in four threshold intensities: two for increasing and two for decreasing pump intensities. When the intensity of the four pumps increases, the regions directly pumped by the lasers jump from the lower to the upper branch of the bistability curve (first threshold). At this point, since polaritons have a finite lifetime, the central region between 


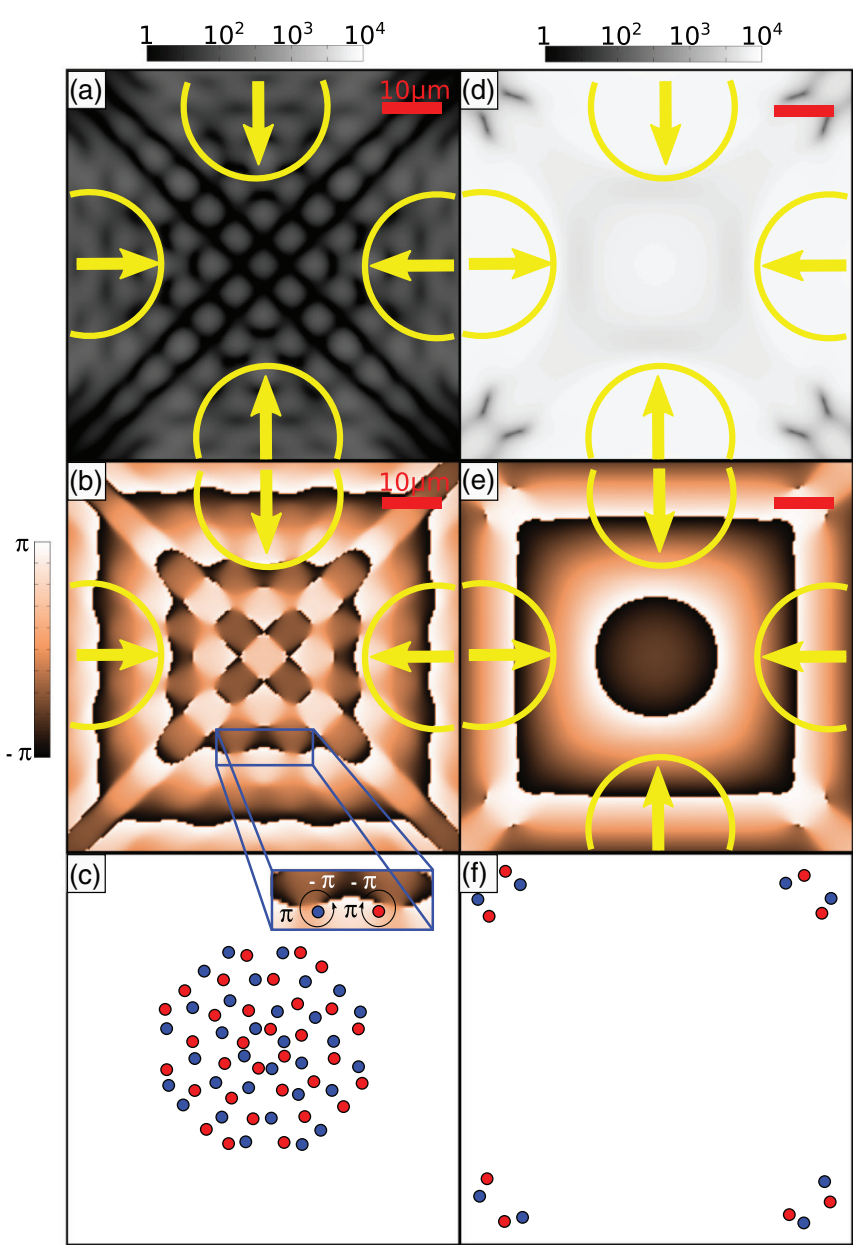

FIG. 1. (Color online) Numerical real-space emission intensity, phase, and vortex distribution in the low and high density regimes. The four polariton ensembles are generated by pumps at $k_{\text {pump }}=0.7 \mu \mathrm{m}^{-1}$ in different directions (yellow arrows) with $\omega_{\text {pump }}=-2.25 \mathrm{meV}$. (a) Real-space image in the low density regime with pump intensity $f_{p}=0.07 f_{\text {th }}$ : A square interference pattern with a unit cell of about $9 \mu \mathrm{m}$ is formed. (d) Real-space image in the high density regime $f_{p}=3.33 f_{\text {th }}$. (b), (e) Phases corresponding to (a) and (d) showing an array of vortices and antivortices (b) and no phase modulations (e). (c), (f) Vortice (red) and antivortice (blue) distributions in real space corresponding to phase diagrams (b)-(e).

the four laser spots is still in the lower part of the bistability curve. As the pump intensity is further increased, the central region also eventually jumps from the lower to the upper branch (second threshold). This threshold corresponds to the intensity $f_{\text {th }}$ at which the V-AV pairs disappear. Similarly, one can observe two thresholds for decreasing pump intensity.

Figures 2(a)-2(c) [Figs. 2(d)-2(f)] represent the polariton distribution (phase) for increasing pump intensity from just below to just above $f_{\text {th }}$. In Fig. 2(a) $\left(f_{p}=0.66 f_{\text {th }}\right)$ some remainder of the interference pattern of Fig. 1(a) is still visible, together with four dark segments surrounding the center of the image that correspond to four V-AV pairs. In this plot of the intensity distribution of each $\mathrm{V}$-AV pair resembles a straight dark segment rather than as two separated vortices because the core of the vortex is extremely close to the core of the antivortex. The fact that these four dark segments correspond

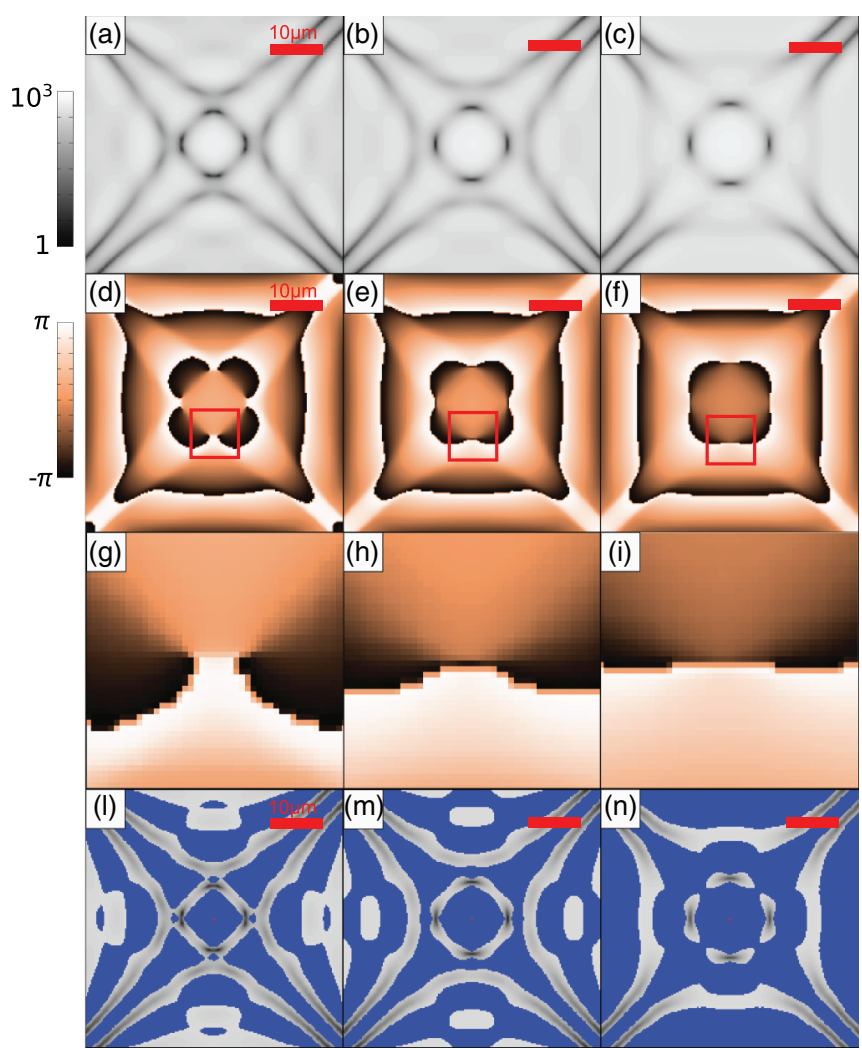

FIG. 2. (Color online) (a)-(c) Numerical real-space emission intensity for three different pump intensities. (d)-(f) Real-space phase diagrams corresponding to the pumping condition of (a)-(c). (g)-(i) Enlargement of the red squared area in (d)-(f). (l)-(n) Mach charts corresponding to (a)-(c): Where the fluid is supersonic, the Mach chart shows the real-space emission intensity at that point, and when the fluid is subsonic, the Mach chart is blue. A V-AV pair is clearly visible in (g), $\mathrm{V}$ and $\mathrm{AV}$ have almost merged in (h), and the vortex pair is no longer present in (i). The pump parameters are the same as in Fig. 1 and the pump intensities (from left to right) are $f_{p}=0.66 f_{\text {th }}$, $0.8 f_{\text {th }}$, and $1.0 f_{\text {th. }}$.

to $\mathrm{V}$-AV pairs is confirmed by the phase distribution of Fig. 2(d), where the two phase rotations of the vortex and of the antivortex are visible, in correspondence to each dark segment of Fig. 2(a). For the sake of clarity the phase distribution of the V-AV pair delimited by the red square in Fig. 2(d) is also reported and enlarged in Fig. $2(\mathrm{~g})$. When the pump intensity is increased [Fig. 2(b) $\left(f_{p}=0.8 f_{\text {th }}\right)$ and Fig. 2(c) $\left.\left(f_{p}=1.0 f_{\text {th }}\right)\right]$, the vortex and the antivortex cores get closer (i.e., the dark segments become shorter) until the four V-AV pairs disappear. For even higher pump intensities the density distribution becomes homogeneous, as in Fig. 1(d). Again, this moving closer and merging of the $\mathrm{V}-\mathrm{AV}$ is confirmed by the corresponding phase distributions [Figs. 2(d)-2(f)], and by the corresponding zoom of the regions delimited by the red squares [Figs. 2(g)-2(i)].

This detailed analysis of the density and phase distributions and of the "on-site" annihilation of V-AV pairs shows that the disappearance of the vortex array is not due to the expulsion of the vortices from the fluid. Therefore, the mechanism lying beneath the V-AV annihilation cannot be ascribed to a simple renormalization of the lower-polariton branch. First, the effect 
of the renormalization is to decrease the wave vector of the injected polaritons and correspondingly to increase the size of the interference pattern of the array, therefore leading to the expulsion of vortices from the fluid rather than the observed merging. Second, it can be seen in Fig. 2(c) that when V-AV annihilation takes place, part of the interference pattern is still visible. Third, vortices and antivortices can exist in a fluid at rest, so the renormalization of the lower-polariton branch cannot justify per se the disappearance of the array.

Moreover, since all the plots show the system steady state for a given pump intensity, the fact that vortices coexist with antivortices means that their position is the result of a tradeoff between vortex-vortex and vortex-antivortex interactions, the direction of the polariton flow, and the relative phase of the four pumps. In other words, this means that vortices and antivortices are not completely free to move in the system and therefore $\mathrm{V}-\mathrm{AV}$ annihilation cannot be simply ascribed to a $\mathrm{V}-\mathrm{AV}$ attraction. In fact, if vortices and antivortices are completely free to move, due to their mutual attraction they must always annihilate, and the only possible steady state must be completely free of vortex dislocations, independently from the intensity of the laser pumps. Finally, it is worth noting that due to the choice of the continuous-wave resonant pumping setup, no exciton reservoir is present in the system and all polaritons have the same energy, therefore no trapping mechanism can be advocated to explain this annihilation as in Ref. [18].

To have physical insights into the mechanism lying beneath $\mathrm{V}-\mathrm{AV}$ annihilation it is useful to study the Mach-number charts in Figs. 2(1)-2(n), where blue regions correspond to a subsonic character of the fluid and gray regions correspond to a supersonic character. Clearly, as the pump intensity is increased (from left to right), the polariton density increases and the regions where the fluid is subsonic it becomes wider and wider. This spreading of the subsonic regions rearranges the polariton flow, therefore changing the steady state position of vortices and antivortices until, when the pump threshold $f_{\text {th }}$ is reached, the vortices and antivortices annihilate. This mechanism can also be understood by observing that vortices cannot enter subsonic regions since they are intrinsically related to regions where the fluid is supersonic, because at the center of their core the fluid density vanishes while the fluid velocity does not, and because a subsonic fluid tends to avoid strong phase modulations [27]. Since in our system the subsonic regions surround the V-AV distribution, when the pump intensity changes, the polariton flows rearrange, letting vortices overlap and annihilate with antivortices. This is different from the case of atomic Bose-Einstein condensates where a single vortex cannot be destroyed by the superfluid character of a quantum fluid since the total angular momentum of the system must be conserved. Here, polariton-polariton interactions can affect the fluid distribution and make a vortex overlap with an antivortex, so that V-AV pairs can be completely suppressed in the fluid.

An additional proof of the correlation between the subsonic character of the fluid and the disappearance of $\mathrm{V}-\mathrm{AV}$ pairs is given by the fact that higher sound velocities (i.e., higherpolariton densities) are needed to destroy the array when polaritons have higher velocities. Figures 3(a)-3(c) show the real-space distribution of the photonic field for three different

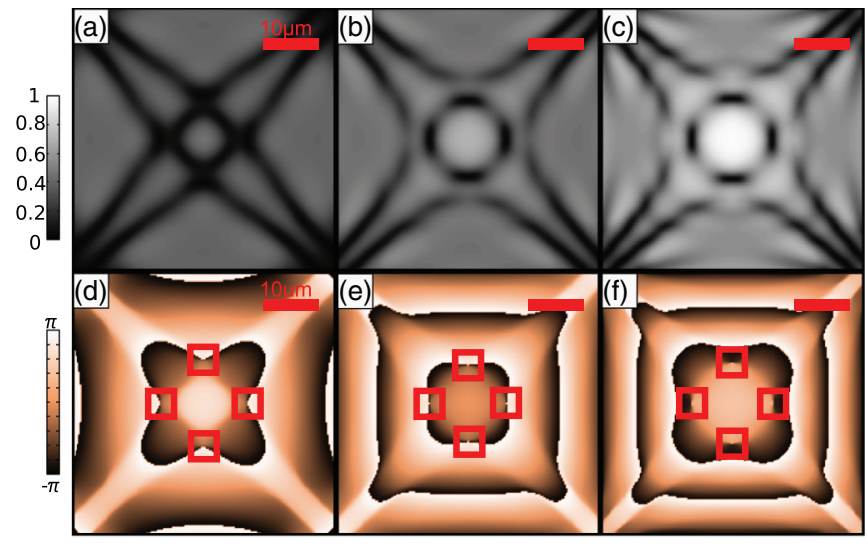

FIG. 3. (Color online) (a)-(c) Numerical real-space emission intensity (in linear grayscale). (d)-(f) Real-space phase diagram (color scale) corresponding to (a)-(c). The red squares indicate the regions where vortex and antivortex pairs have been annihilated. The plots correspond to pumps with increasing in-plane momenta from left to right: $k_{\text {pump }}=0.5,0.7$, and $0.9 \mu \mathrm{m}^{-1}$. In each case the pump intensity is chosen in order to drive the system at the threshold at which V-AV annihilation occurs $\left[f_{p}(\mathrm{~b})=4.33 f_{p}(\mathrm{a}), f_{p}(\mathrm{c})=8.66 f_{p}(\mathrm{a})\right]$.

increasing velocities of the injected polaritons $\left(k_{\text {pump }}=0.5\right.$, 0.7 , and $0.9 \mu \mathrm{m}^{-1}$ ) and a pump intensity corresponding to the threshold $\left(f_{p}=f_{\text {th }}\right.$ ) where V-AV pairs disappear. We find that the polariton density, and therefore the sound velocity, at which V-AV pairs annihilate is higher when the velocity of the injected polaritons is higher [see Figs. 3(d)-3(f), where no phase cut corresponding to a $\mathrm{V}$-AV pair can be observed in the four regions delimited by the red squares]. This confirms that polariton-polariton interactions, causing the widening of the subsonic regions and the consequent rearrangement of the polariton flows, induce the disappearance of $\mathrm{V}-\mathrm{AV}$ pairs.

\section{CONCLUSIONS}

We have investigated the washing out of vortex-antivortex lattices in exciton-polariton systems as a function of the polariton density. Our detailed analysis shows that V-AV annihilation and the washing out of the vortex lattice is due to polaritonpolariton interactions that, through the renormalization of the lower-polariton branch, induce the widening of the regions where the fluid is subsonic as the pump intensity is increased. This widening induces a modification in the polariton flows, therefore changing the steady state position of the vortices and antivortices until a threshold pump intensity is reached at which $\mathrm{V}$-AV merging takes place. The complete washing out of the V-AV array can take place because in our system the number of vortices is equal to the number of antivortices, i.e., the system has zero angular momentum. Our analysis, when applied to a system with net angular momentum, could open a way to the study of vortex-vortex interactions.

\section{ACKNOWLEDGMENTS}

We would like to thank Prof. C. Tejedor for the use of his computational facilities and I. Carusotto and F. M. Marchetti for useful discussions. This work has been partially funded by the Quandyde project of the ANR France, by the POLATOM 
ESF Research Network Program, and by the CLERMONT4 Network Program. M.H.S was supported by the EPSRC
(Grants No. EP/I028900/2 and No. EP/K003623/2) D.B. and D.S. acknowledge funding from the ERC project PLAFLOW.
[1] A. S. Desyatnikov, Y. S. Kivshar, and L. Torner, in Progress in Optics, edited by E. Wolf (Elsevier, Amsterdam, 2005), Vol. 47, pp. 291-391.

[2] U. Essmann and H. Träuble, Phys. Lett. A 24, 526 (1967).

[3] E. J. Yarmchuk, M. J. V. Gordon, and R. E. Packard, Phys. Rev. Lett. 43, 214 (1979).

[4] J. Scheuer and M. Orenstein, Science 285, 230 (1999).

[5] K. W. Madison, F. Chevy, W. Wohlleben, and J. Dalibard, Phys. Rev. Lett. 84, 806 (2000).

[6] J. Denschlag, J. E. Simsarian, D. L. Feder, C. W. Clark, L. A. Collins, J. Cubizolles, L. Deng, E. W. Hagley, K. Helmerson, W. P. Reinhardt, S. L. Rolston, B. I. Schneider, and W. D. Phillips, Science 287, 97 (2000).

[7] L. Khaykovich, F. Schreck, G. Ferrari, T. Bourdel, J. Cubizolles, L. D. Carr, Y. Castin, and C. Salomon, Science 296, 1290 (2002).

[8] K. G. Lagoudakis, M. Wouters, M. Richard, A. Baas, I. Carusotto, R. André, L. S. Dang, and B. Deveaud-Plédran, Nat. Phys. 4, 706 (2008).

[9] K. G. Lagoudakis, T. Ostatnický, A. V. Kavokin, Y. G. Rubo, R. André, and B. Deveaud-Plédran, Science 326, 974 (2009).

[10] H. Flayac, I. A. Shelykh, D. D. Solnyshkov, and G. Malpuech, Phys. Rev. B 81, 045318 (2010).

[11] G. Roumpos, M. D. Fraser, A. Löffler, S. Höfling, A. Forchel, and Y. Yamamoto, Nat. Phys. 7, 129 (2011).

[12] G. Nardin, G. Grosso, Y. Léger, B. Pitka, F. Morier-Genoud, and B. Deveaud-Plédran, Nat. Phys. 7, 635 (2011).

[13] G. Tosi, F. M. Marchetti, D. Sanvitto, C. Antón, M. H. Szymańska, A. Berceanu, C. Tejedor, L. Marrucci, A. Lemaître, J. Bloch, and L. Viña, Phys. Rev. Lett. 107, 036401 (2011).
[14] D. Sanvitto, S. Pigeon, A. Amo, D. Ballarini, M. D. Giorgi, I. Carusotto, R. Hivet, F. Pisanello, V. G. Sala, P. S. S. Guimaraes, R. Houdré, E. Giacobino, C. Ciuti, A. Bramati, and G. Gigli, Nat. Photonics 5, 610 (2011).

[15] A. V. Gorbach, R. Hartley, and D. V. Skryabin, Phys. Rev. Lett. 104, 213903 (2010).

[16] J. Keeling and N. G. Berloff, Phys. Rev. Lett. 100, 250401 (2008).

[17] G. Tosi, G. Christmann, N. G. Berloff, P. Tsotsis, T. Gao, Z. Hatzopoulos, P. G. Savvidis, and J. J. Baumberg, Nat. Commun. 3, 1243 (2012).

[18] P. Cristofolini, A. Dreismann, G. Christmann, G. Franchetti, N. G. Berloff, P. Tsotsis, Z. Hatzopoulos, P. G. Savvidis, and J. J. Baumberg, Phys. Rev. Lett. 110, 186403 (2013).

[19] K. Kusudo, N. Y. Kim, A. Löffler, S. Höfling, A. Forchel, and Y. Yamamoto, Phys. Rev. B 87, 214503 (2013).

[20] T. C. H. Liew, Y. G. Rubo, and A. V. Kavokin, Phys. Rev. Lett. 101, 187401 (2008).

[21] R. Hivet, E. Cancellieri, T. Boulier, D. Ballarini, D. Sanvitto, F. M. Marchetti, M. H. Szymanska, C. Ciuti, E. Giacobino, and A. Bramati, Phys. Rev. B 89, 134501 (2014).

[22] I. Carusotto and C. Ciuti, Rev. Mod. Phys. 85, 299 (2013).

[23] M. Wouters, I. Carusotto, and C. Ciuti, Phys. Rev. B 77, 115340 (2008).

[24] C. Ciuti and I. Carusotto, Phys. Status Solidi B 242, 2224 (2005).

[25] F. Liu and G. F. Mazenko, Phys. Rev. B 46, 5963 (1992).

[26] R. Osborne, Philos. Trans. R. Soc., A 186, 123 (1895).

[27] L. D. Landau and E. M. Lifshitz, in Statistical Physics: Part 1, Course of Theoretical Physics, 2nd ed. (Pergamon, Oxford, UK, 1986), Chap. 6. 